\title{
From OPE to chiral perturbation theory in holographic QCD
}

\section{David Greynat*}

Dip. di Scienze Fisiche, Universitá di Napoli "Federico II", Via Cintia, 80126 Napoli, Italia INFN-Sezione di Napoli, Via Cintia, 80126 Napoli, Italia

E-mail: david.greynategmail.com

\section{Luigi Cappiello}

Dip. di Scienze Fisiche, Universitá di Napoli "Federico II", Via Cintia, 80126 Napoli, Italia INFN-Sezione di Napoli, Via Cintia, 80126 Napoli, Italia

E-mail: luigi.cappiello@na.infn.it

\section{Giancarlo D'Ambrosio}

INFN-Sezione di Napoli, Via Cintia, 80126 Napoli, Italia

E-mail: gdambros@na.infn.it

The soft wall model in holographic QCD has Regge trajectories but wrong operator product expansion (OPE) for the two-point vectorial QCD Green function. We modify the dilaton potential to comply with the OPE. We study also the axial two-point function using the same modified dilaton field and an additional scalar field to address chiral symmetry breaking. OPE is recovered adding a boundary term and low energy chiral parameters, $F_{\pi}$ and $L_{10}$, are well described analytically by the model in terms of Regge spacing and QCD condensates. The model nicely supports and extends previous theoretical analyses advocating Digamma function to study QCD two-point functions in different momentum regions.

The 8th International Workshop on Chiral Dynamics, CD2015 ***

29 June 2015 - 03 July 2015

Pisa,Italy

\footnotetext{
*Speaker.
} 


\section{Introduction}

This proceeding is based on our previous work [1]. As matter of fact, one knows partially QCD Green functions on three ranges of energies ( $i$ ) deep Euclidean, where perturbative QCD and OPE expansion methods can be applied, (ii) an intermediate Minkowski region, where resonances are described by Regge trajectories and (iii) the strong interacting, low-energy region, described by chiral perturbation theory $(\chi \mathrm{PT})$ [2]. Unfortunately, global analytic expressions for each of them are unknown due to the complexity of the non-perturbative properties of QCD. It is natural to ask ourself if it is possible to reconstruct an approximate analytic expression satisfying all QCD properties valid in any region. In this approach, we think that Regge trajectories, i.e. a linear growth of the square of the resonance masses like $M_{V}^{2}(n)=\sigma n \mathrm{GeV}^{2}$ for the vectors, were conjectured long ago, before QCD, and very well verified phenomenologically [3] play a crucial role [4, 5, 6, 7].

In the three energy sectors, the AdS/CFT correspondence $[8,9,10]$ has been useful to describe the QCD properties. At low energy, QCD properties like the chiral symmetry breaking $(\chi \mathrm{SB})$ parameters, $F_{\pi}$ and the Gasser-Leutwyler coefficients $L_{i}$ 's of the $\mathscr{O}\left(p^{4}\right)$ chiral Lagrangian have been studied in several five dimensional set-ups. The basic feature of Hard-Wall (HW) models is to simulate confinement by cutting drastically the extradimension of the $\mathrm{AdS}_{5}$ in the IR, producing an infinite spectrum of Kaluza-Klein (KK) states to be identified with vector resonances of increasing masses. $\chi \mathrm{SB}$ in the axial sector is triggered either by a scalar field in the bulk [11, 12] or by appropriate boundary conditions [13]. A recent review of all these models and their relation with the light-front holographic QCD approach is Ref [15].

All these models have a good description of the deep Euclidean region; for instance the correct two point function recover both the partonic log and a pretty well description of low energy QCD, obtaining values of the chiral parameters $F_{\pi}$ and the Gasser-Leutwyler coefficients $L_{i}$ 's which are close to the physical ones. In fact, it was observed that these HW models [11, 12, 13] have the same two point vectorial Green function as the one obtained by Migdal long ago who proposed an ad hoc prescription to perform the analytic continuation of the perturbative deep Euclidean QCD result to the Minkowski region $[17,16]$.

In order to reproduce resonances masses with a Regge spacing, one can consider a 5D model with AdS metric and an additional field, the dilaton [18]. However, in this model it can be shown that the partonic log of the two point vectorial Green function receives $1 / Q^{2}$-corrections differently from what holds in perturbative QCD Operator Product Expansion (OPE) [19, 20, 21, 22]. Nevertheless, we find extremely interesting to have a model where the Regge region would be analytically related to the deep Euclidean region even though with the wrong OPE. This will be exactly our starting point: OPE tells us the correct Green functions in the deep Euclidean in terms of gluon and quarks condensates. Is it possible to modify the dilaton profile, $\phi(z) \mapsto \phi(z)+\delta \phi(z)$, such to comply with QCD requirements in the intermediate (Regge) region and UV (OPE) region? Once this question has been answered in the affirmative, we are naturally led to address another important problem, that does not have a satisfying answer in this "linear confinement" holographic approach [18]: how to implement the effects of chiral symmetry breaking into our model. In fact, implementing the correct OPE and mass spectrum for the axial sector, we will propose our post-diction of $F_{\pi}$ and $L_{10}$ (one of the the chiral $\mathscr{O}\left(p^{4}\right)$ coefficients) in terms of our input parameters, i.e. the Regge spacing and the QCD condensates. Our model, beside being a novel proposal for holographic 
QCD, links naturally to previous 4D QCD work where a phenomenological matching between low energy and OPE was realised through Regge theory (or Veneziano model) [2, 23, 24, 5, 25, 26, 27].

\section{Construction of the model}

The Soft-Wall model is a five dimensional model where the additional coordinate, $z$, has the range $0<z<\infty$ and background fields consist in a five-dimensional AdS metric and a dilaton field $\Phi(z)$. The AdS metric is written as

$$
g_{M N} \mathrm{~d} x^{M} \mathrm{~d} x^{N}=\frac{1}{z^{2}}\left(\eta_{\mu \nu} \mathrm{d} x^{\mu} \mathrm{d} x^{\nu}-\mathrm{d} z^{2}\right)
$$

where $\eta_{\mu v}=\operatorname{Diag}(1,-1,-1,-1)$, the Greek indices $\mu, v=(0,1,2,3)$ referring to the usual 4dimensions, and the capital Latin ones $M, N=(0,1,2,3, z)$ to the 5 dimensions. It was shown, in [18], that with the choice of a quadratic profile of the dilaton field

$$
\Phi(z) \doteq \kappa^{2} z^{2}
$$

the spectrum of vector resonances followed a Regge trajectory.

Our model is based on the following five dimensional action,

$$
\begin{aligned}
S_{5}=\int \mathrm{d}^{4} x \int_{0}^{\infty} \mathrm{d} z w_{0}(z) \operatorname{Tr}\left\{g^{M N}\left(D_{M} \mathbb{X}\right)^{\dagger}\left(D_{N} \mathbb{X}\right)\right. & -m^{2} \mathbb{X}^{2} \\
& \left.-\frac{1}{4 g_{5}^{2}} g_{M N} g_{R S}\left(\mathbb{F}_{V}^{M R} \mathbb{F}_{V}^{N S}+\mathbb{F}_{A}^{M R} \mathbb{F}_{A}^{N S}\right)\right\},
\end{aligned}
$$

since the generic field strength (for vector and identical for axial vector) is $\mathbb{F}_{V M N}=\partial_{M} \mathbb{V}_{M}$ $\partial_{N} \mathbb{V}_{M}-i\left[\mathbb{V}_{M}, \mathbb{V}_{N}\right]$ and $g_{5}^{2}=12 \pi^{2} / N_{c}$ is the 5D coupling constant where $N_{c}$ is the number of colors of $\mathrm{QCD}, D^{M} \mathbb{X}=\partial^{M} \mathbb{X}-i\left[\mathbb{V}^{M}, \mathbb{X}\right]-i\left\{\mathbb{A}^{M}, \mathbb{X}\right\}$, where the 5D fields $\mathbb{V}_{M}, \mathbb{A}_{M}$ and $\mathbb{X}$ are respectively the vectorial, axial and scalar fields. We defined also,

$$
w_{0}(z) \doteq \frac{\mathrm{e}^{-\Phi(z)}}{z}=\frac{\mathrm{e}^{-\kappa^{2} z^{2}}}{z} .
$$

\subsection{The vectorial sector}

Let's consider first the vectorial sector. We shall work in the axial gauge $\mathbb{V}_{z}=0$. The AdS/CFT correspondence prescribes that the boundary value of the $5 \mathrm{D}$ gauge field $\mathbb{V}_{\mu}$ has to be identified with the classical source $\mathrm{v}_{\mu}$ coupled to the the 4-dimensional vectorial current $J_{V \mu}^{a}=: \bar{q} \gamma_{\mu} t^{a} q$ :,

$$
\lim _{z \rightarrow 0} \mathbb{V}_{\mu, z}^{a}(x, z)=\mathrm{v}_{\mu}^{a}(x) .
$$

The corresponding equation of motion for the gauge field, derived from the Lagrangian (2.3), is more easily written in terms of the 4-dimensional Fourier transform $f_{V}\left(-q^{2}, z\right)$ of the field $\mathbb{V}_{M}$,

$$
f_{V}\left(-q^{2}, z\right) \hat{\mathrm{v}}_{\mu}(q) \doteq \int \mathrm{d}^{4} x \mathrm{e}^{-i q \cdot x} \mathbb{V}_{\mu, 0}(x, z)
$$


where $\hat{v}_{\mu}$ is the Fourier transform of the source $v_{\mu}$. Using gauge invariance, one may assume the $\hat{\mathrm{v}}_{\mu}$ to be transverse and one obtains

$$
\partial_{z}^{2} f_{V}+\partial_{z}\left[\ln w_{0}(z)\right] \partial_{z} f_{V}+q^{2} f_{V}=0
$$

with the boundary conditions $(2.5)$

$$
f_{V}\left(-q^{2}, 0\right)=1 \quad \text { and } \quad f_{V}\left(-q^{2}, \infty\right)=0 .
$$

In this context, the vector-vector correlator $\Pi_{V}$,

$$
i \int \mathrm{d}^{4} x \mathrm{e}^{-i q \cdot x}\left\langle J_{V \mu}^{a}(x) J_{V v}^{b}(0)\right\rangle=\delta^{a b}\left(q_{\mu} q_{v}-q^{2} \eta_{\mu v}\right) \Pi_{V}\left(-q^{2}\right) .
$$

where $q$ is the $4 \mathrm{D}$ momentum and the current $J_{V \mu}^{a}$ is the one defined before equation (2.5) is extracted from the 5D expressions by taking the limit (with the Euclidean momentum $Q^{2} \doteq-q^{2}$ )

$$
Q^{2} \Pi_{V}\left(Q^{2}\right)=\frac{1}{g_{5}^{2}} \lim _{z \rightarrow 0} w_{0}(z) f_{V}\left(Q^{2}, z\right) \partial_{z} f_{V}\left(Q^{2}, z\right) .
$$

Our main concern is that the two-point function $\Pi_{V}$ coming from the model built in $5 \mathrm{D}$ has the following properties:

(i) The progression of its poles in the Minkowski region (the resonances) follows a Regge trajectory.

In the Large- $\mathrm{N}_{c}$ limit of QCD, the two-point function $\Pi_{V}$ could be written as a sum over an infinite set of stable vector resonances $[29,30]$

$$
\Pi_{V}\left(Q^{2}\right)=\sum_{n=0}^{\infty} \frac{F_{V}(n)^{2}}{Q^{2}+M_{V}(n)^{2}},
$$

where $F_{V}(n)$ are named decay constants and the $M_{V}(n)$ are the masses associated to the resonances of the vectorial channel: $\rho, \rho^{\prime}, \rho^{\prime \prime}, \ldots$. We assume that these resonances follow, in a first approximation, a Regge progression [3],

$$
M_{V}(n)^{2} \underset{n \rightarrow \infty}{\sim} \sigma n
$$

where the integer $n$ is the radial excitation number and $\sigma$ is related to the confining string tension as explained in [18] and we can evaluate $\sigma \approx 0.90 \mathrm{GeV}^{2}$ from [31, 32].

(ii) $\Pi_{V}$ has the correct OPE. One of the very well-known properties of this two point function is its OPE [33],

$$
\Pi_{V}\left(Q^{2}\right) \underset{Q^{2} \rightarrow \infty}{\sim} \frac{1}{2} \frac{N_{c}}{12 \pi^{2}} \ln \left(\frac{\Lambda_{V}^{2}}{Q^{2}}\right)+\left\langle\mathscr{O}_{2}\right\rangle \frac{1}{Q^{2}}+\left\langle\mathscr{O}_{4}\right\rangle \frac{1}{Q^{4}}+\left\langle\mathscr{O}_{6}\right\rangle_{V} \frac{1}{Q^{6}}
$$

where in the large- $\mathrm{N}_{c}$ limit the coefficients of the OPE are given by

$$
\left\{\begin{array}{l}
\left\langle\mathscr{O}_{2}\right\rangle=0 \\
\left\langle\mathscr{O}_{4}\right\rangle=\frac{1}{2} \frac{1}{12 \pi} \alpha_{s}\left\langle G^{2}\right\rangle \\
\left\langle\mathscr{O}_{6}\right\rangle_{V}=\frac{1}{2}\left(-\frac{28 \pi}{9}\right) \alpha_{s}\langle\bar{\psi} \psi\rangle^{2}
\end{array},\right.
$$

here $\alpha_{s}$ stands for the strong coupling constant, $\left\langle G^{2}\right\rangle$ for the gluon condensate, and $\langle\bar{\psi} \psi\rangle$ for the quark condensate. 
If one solves the differential equation (2.7) with the boundary conditions (2.8), one obtains

$$
Q^{2} \Pi_{V}^{(0)}\left(Q^{2}\right)=-\frac{2 \kappa^{2}}{g_{5}^{2}}\left(\frac{Q^{2}}{4 \kappa^{2}}\right)\left[\gamma_{E}+\psi\left(\frac{Q^{2}}{4 \kappa^{2}}+1\right)\right]
$$

which gives the following OPE,

$$
\Pi_{V}^{(0)}\left(Q^{2}\right) \underset{Q^{2} \rightarrow \infty}{\sim} \frac{1}{2 g_{5}^{2}} \ln \left(\frac{4 \kappa^{2} \mathrm{e}^{-\gamma_{E}}}{Q^{2}}\right)+\frac{\kappa^{2}}{g_{5}^{2}} \frac{1}{Q^{2}} .
$$

Unfortunately, this solution (2.15) does not satisfy the full OPE (2.13) of the vectorial twopoint function. In order to cure this problem, we shall assume that the effects of the OPE on the vector current two-point functions in QCD can be encoded in a new profile for the dilaton field of the SW model. More explicitly, we assume that OPE is related to the behaviour of the dilaton profile around the UV boundary, $z=0$ of the $5 \mathrm{D}$, while keeping the metric to have the AdS form (2.1). Moreover, as we want to keep the Regge behaviour induced by the dilaton profile $\Phi(z)$, we are led to modify the original quadratic profile of the SW dilaton by adding new terms which we collect in a function $B$,

$$
\Phi(z) \longmapsto \Phi(z)+B(z)
$$

We assume that the function $B$ can be represented for all $z$ by a polynomial of degree $2 K$ (with no constant term),

$$
B(z)=\sum_{k=1}^{K} \frac{b_{2 k}}{2 k} z^{2 k}
$$

We prove in [1] then that the equation of motion (2.7) becomes

$$
\partial_{z}^{2} f_{V}+\partial_{z}[\ln w(z)] \partial_{z} f_{V}-Q^{2} f_{V}=0
$$

with $w(z) \doteq w_{0}(z) \mathrm{e}^{-B(z)}$ and can be decomposed into a solvable hierarchical system of differential equations order by order.

This general solution drives to an expression for the vectorial correlator,

$$
Q^{2} \Pi_{V}\left(Q^{2}\right)=\sum_{k} \mathscr{P}_{k}\left(\frac{Q^{2}}{4 \kappa^{2}}\right) \psi^{(k)}\left(\frac{Q^{2}}{4 \kappa^{2}}\right),
$$

where, respectively, $\mathscr{P}_{k}$ are polynomials and $\psi^{(k)}$ is the $k^{\text {th }}$ derivative of the Digamma $\psi$ function defined as the logarithmic derivative of the Euler's $\Gamma$ function. The coefficients of $\mathscr{P}_{k}$ depend only on $\kappa^{2}$ and the coefficients $b_{k}$ of the dilaton (2.18).

This solution fulfils perfectly our two requirements $(i)$ and (ii). Indeed, the Digamma function and its derivatives in (2.20) have poles only at all negative integers $(-n)$,

$$
\frac{-q^{2}}{4 \kappa^{2}}=-n
$$

so the usual Regge spectrum is verified,

$$
M(n)^{2}=4 \kappa^{2} n=\sigma n,
$$

fixing $\kappa^{2}=\sigma / 4$. 
On the other hand, performing the expansion at $Q^{2} \rightarrow \infty$ of (2.20), we prove in [1] that we identify

$$
b_{2 k}=\left\{\begin{array}{ll}
-2 \kappa^{2} & k=1 \\
g_{5}^{2} \frac{2}{\sqrt{\pi}} \frac{\Gamma\left(\frac{1}{2}+k\right)}{\Gamma(k) \Gamma^{2}(k+1)}\left\langle\mathscr{O}_{2 k}\right\rangle_{V} & k>1
\end{array},\right.
$$

in order to reproduce the OPE of the vectorial two-point function (2.13). We have also shown in [1] that we can always see this modification of the dilatonic profile $B(z)$ as a perturbation. Indeed, one can introduce an artificial control parameter $\theta$ which allows a formal perturbative expansion in a region where the range of parameters is valid. Moreover, $\theta$ allows to see explicitly the sub-leading Regge trajectories corrections and the associated amplitude modifications.

\subsection{Axial sector}

In the previous section, we have presented a SW model with a deformed dilaton profile which describes Regge theory and allows us to obtain the correct OPE of the vectorial two-point function. The natural question is now if it is possible to do a similar construction for the axial correlator. The problem to recover the right axial spectrum and the right axial OPE is more complicated if we assume, as usual, that the coupling of the 5D vector and axial vector gauge field to the metric and the dilaton is the same. Actually, under these assumptions, the form of the dilaton profile is already fixed by the requirements of a correct OPE of the vector two-point function, with the coefficients $b_{k}$ identified to the vectorial OPE coefficients (2.23). So we introduce a 5D scalar field $\mathbb{X}(x, z)$ in (2.3) to reproduce the QCD patterns of chiral symmetry breaking as in [11, 12]. They showed that by taking

$$
\mathbb{X}^{a} \doteq \frac{v(z)}{2} \mathbb{I}^{a}
$$

the equation of motion for the Fourier transform over the $4 \mathrm{D}$ space of the axial field $\mathbb{A}, f_{A}\left(-q^{2}, z\right)$, becomes

$$
\partial_{z}^{2} f_{A}+\partial_{z}[\ln w(z)] \partial_{z} f_{A}-Q^{2} f_{A}=g_{5}^{2}\left(\frac{v(z)}{z}\right)^{2} f_{A},
$$

while the equation of motion for the vector field remains unchanged.

In this approach, chiral symmetry is broken by the 5D scalar field, and in particular by a non vanishing profile $v(z)$. The form of $v(z)$ near the origin $z \sim 0$ is dictated in the AdS/CFT correspondence by asking the field $\mathbb{X}$ to be dual to the bilinear quark $q \bar{q}$ operator, whose non vanishing VEV is responsible for spontaneous $\chi \mathrm{SB}$ in QCD. In the following, we shall assume for the dilaton profile $B(z)$ the one obtained from the OPE coefficients of the vectorial fields and then use a suitable form of the profile $v(z)$ to encode the properties of the axial sector:

- The axial spectrum contains a pion pole at $q^{2}=0$ and a Regge spectrum starting at $q^{2}=M_{a_{1}}^{2}$ with the same spacing than the vectorial spectrum.

- The axial OPE has the following expression

$$
\Pi_{A}\left(Q^{2}\right) \underset{Q^{2} \rightarrow \infty}{\sim} \frac{N_{c}}{24 \pi^{2}} \ln \left(\frac{\Lambda_{A}^{2}}{Q^{2}}\right)+\left\langle\mathscr{O}_{2}\right\rangle \frac{1}{Q^{2}}+\left\langle\mathscr{O}_{4}\right\rangle \frac{1}{Q^{4}}+\left\langle\mathscr{O}_{6}\right\rangle_{A} \frac{1}{Q^{6}}
$$


and

$$
\left\langle\mathscr{O}_{6}\right\rangle_{A}=-\frac{11}{7}\left\langle\mathscr{O}_{6}\right\rangle_{V}
$$

with the same definitions than in (2.14).

In order to satisfy the axial spectral properties, we make the following first ansatz for the contribution due to the scalar profile in (2.25):

$$
\left(\frac{v(z)}{z}\right)^{2}=\beta_{0}+\beta^{*} z \delta(z)
$$

Let focus first on the Regge progression of the axial spectrum. We notice that, phenomenologically, taking the first axial pole at $q^{2}=M_{a_{1}}^{2} \simeq 2 M_{\rho}^{2} \simeq 2 \sigma$ is quite an acceptable approximation for the axial spectrum. Then our prescription to obtain the axial spectrum from the vectorial one is to perform a "shift" over the vectorial spectrum like $Q^{2} \rightarrow Q^{2}+4 \kappa^{2}$. This fixes directly in (2.25)

$$
g_{5}^{2} \beta_{0}=4 \kappa^{2}=\sigma
$$

The full axial spectrum is not yet realised, indeed, since the axial spectrum is a shifted version of the vectorial spectrum, it does not contain yet any pion pole and moreover on the Euclidean side, this shift implies a modifcation of the OPE as

$$
\Pi_{A}\left(Q^{2}\right) \underset{Q^{2} \rightarrow \infty}{\sim} \frac{1}{2 g_{5}^{2}} \ln \left(\frac{4 \kappa^{2} \mathrm{e}^{-\gamma_{E}}}{Q^{2}}\right)+\frac{2 \kappa^{2}}{g_{5}^{2}} \frac{1}{Q^{2}},
$$

where absence of dimension two operator in the axial OPE is violated.

The introduction of the Dirac delta function in (2.28) has the nice properties to cure this two problems at the same time. This term in (2.25) generates only one exact contribution $-\beta^{*} / Q^{2}$ (for any $Q^{2}$ ) such that the pion pole appears naturally and modifying the OPE as

$$
\Pi_{A}\left(Q^{2}\right) \underset{Q^{2} \rightarrow \infty}{\sim} \frac{1}{2 g_{5}^{2}} \ln \left(\frac{4 \kappa^{2} \mathrm{e}^{-\gamma_{E}}}{Q^{2}}\right)+\left(\frac{2 \kappa^{2}}{g_{5}^{2}}-\beta^{*}\right) \frac{1}{Q^{2}},
$$

then by taking

$$
\beta^{*}=\frac{2 \kappa^{2}}{g_{5}^{2}}
$$

the axial OPE properties is satisfied again.

The generation of the rest of the axial OPE terms derives exactly from the same procedure used in the vectorial section provided that we add two other terms in the expression (2.28) such as

$$
\left(\frac{v(z)}{z}\right)^{2}=\beta_{0}+\beta^{*} z \delta(z)+\beta_{2} z^{2}+\beta_{4} z^{4} .
$$

With slight modifications, the iterative method we used to obtain the corrections to the vector two-point function, can be applied to the axial case too. All the details can be found in [1]. Then 
one has by identification: $\Lambda_{A}^{2}=4 \kappa^{2} \mathrm{e}^{-\gamma_{E}}=\Lambda_{V}^{2}$, the coefficients $\beta_{2}$ and $\beta_{4}$ are fixed by matching with QCD axial OPE (2.26),(2.27) :

$$
\left\{\begin{array}{l}
\beta_{2}=-\frac{6 \kappa^{4}}{g_{5}^{2}} \\
\beta_{4}=-\frac{10 \kappa^{2}}{3 g_{5}^{2}}-5 \kappa^{2}\left\langle\mathscr{O}_{4}\right\rangle+\frac{45}{28}\left\langle\mathscr{O}_{6}\right\rangle_{V} .
\end{array}\right.
$$

The polynomial part of the scalar profile $v(z)$ is

$$
\begin{aligned}
v(z) & =z \sqrt{\beta_{0}+\beta_{2} z^{2}+\beta_{4} z^{4}} \\
& \underset{z \rightarrow 0}{\sim} \frac{2 \kappa}{g_{5}} z-\frac{3 \kappa^{3}}{2 g_{5}} z^{3}+\left(-\frac{67 \kappa^{5}}{48 g_{5}}-\frac{5 g_{5} \kappa}{4}\left\langle\mathscr{O}_{4}\right\rangle+\frac{45 g_{5}}{112 \kappa}\left\langle\mathscr{O}_{6}\right\rangle_{V}\right) z^{5},
\end{aligned}
$$

where the first two terms, which are the leading terms near $z=0$, are exactly the ones of a scalar field dual to the bilinear quark $q \bar{q}$ operator, i.e. the one required in Ref. [28] and compatible with the approach in [34].

\section{Analytic continuation in the chiral sector: the left-right correlator}

We have explicitly shown how to implement the constraints on an axial and vectorial two-point functions coming from two different regions in the $q^{2}$-plane: the deep Euclidean region where we reproduce the matching with the OPE of QCD, and the Minkowski region where the two-point function have poles following Regge trajectories. Having built explicit expressions for $\Pi_{V}$ and $\Pi_{A}$ valid on the whole complex plane we now turn to the analysis of their prediction for chiral quantities defined at low $Q^{2}$.

Since we are now interested to the chiral sector, i.e. the low $Q^{2}$ expansions, it is more pertinent to consider the $\Pi_{L R}$ correlator,

$$
\Pi_{L R}\left(Q^{2}\right)=\frac{1}{2}\left(\Pi_{V}\left(Q^{2}\right)-\Pi_{A}\left(Q^{2}\right)\right) .
$$

which is an order parameter of the chiral symmetry breaking mechanism in QCD.

The low $Q^{2}$ limit can be obtained by the analytic continuation of our expressions for the axial and vectorial two-point function, this allows us to extract from $\Pi_{L R}$ for instance the following chiral constants

$$
\begin{aligned}
F_{\pi}^{2} & =2 \operatorname{Res}\left[\Pi_{L R}\left(Q^{2}\right), 0\right] \\
L_{10} & =\left.\frac{1}{2} \frac{\mathrm{d}}{\mathrm{d} Q^{2}}\left[Q^{2} \Pi_{L R}\left(Q^{2}\right)\right]\right|_{Q^{2}=0} .
\end{aligned}
$$

From our analytic expressions for the vectorial and axial correlator, one obtains the following analytic expressions for $F_{\pi}^{2}$ and $L_{10}$,

$$
F_{\pi}^{2}=\frac{N_{c} \kappa^{2}\left(180 \zeta(3)+191-41 \pi^{2}\right)}{72 \pi^{2}}+\frac{5\left(\pi^{2}-10\right)}{2} \frac{\left\langle\mathscr{O}_{4}\right\rangle}{\kappa^{2}}-\frac{45\left(\pi^{2}-10\right)}{56} \frac{\left\langle\mathscr{O}_{6}\right\rangle_{V}}{\kappa^{4}}
$$


using $N_{c}=3, \kappa=\sqrt{1.43 / 4} \mathrm{GeV} \simeq 0.6 \mathrm{GeV}$ and the values of the condensates $\left\langle\mathscr{O}_{4}\right\rangle=(-0.635 \pm$ $0.04) \cdot 10^{-3} \mathrm{GeV}^{4}$ and $\left\langle\mathscr{O}_{6}\right\rangle_{V}=(14 \pm 3) \cdot 10^{-4} \mathrm{GeV}^{6}$ from [5], we obtain

$$
F_{\pi} \simeq \sqrt{4099.9+579+1147.8} \mathrm{MeV} \simeq 76( \pm 3)_{\mathrm{ext} .} \mathrm{MeV},
$$

the error in (3.5) are coming from the errors quoted for $\sigma$ and the condensates.

The expression for $L_{10}$ is

$$
\begin{aligned}
L_{10}=\frac{N_{c}\left(8010 \zeta(3)+495-585 \pi^{2}-46 \pi^{4}\right)}{8640 \pi^{2}} & \\
+ & +\frac{-72 \zeta(3)-12+11 \pi^{2}}{64} \frac{\left\langle\mathscr{O}_{4}\right\rangle}{\kappa^{4}}+\frac{5\left[5216 \zeta(3)+67-33 \pi^{2}\right]}{1792} \frac{\left\langle\mathscr{O}_{6}\right\rangle_{V}}{\kappa^{6}},
\end{aligned}
$$

then with the same numerical values used for the evaluation of $F_{\pi}$,

$$
10^{3} L_{10} \simeq-4.6-0.8+0.1 \simeq-5.3( \pm 1)_{\mathrm{ext} .},
$$

the error in (3.7) are coming from the errors quoted for $\sigma$ and the condensates ${ }^{1}$.

The values of $F_{\pi}$ and $L_{10}$ are quite reasonable face with our model hypothesis, if one admits the usual 30\% error coming from Large- $\mathrm{N}_{c}$ QCD limit, compared to the range of variation of $F_{\pi}$ in the chiral limit, $66<F_{\pi}<84 \mathrm{MeV}$, and compared to the value $10^{3} L_{10}=-5.3 \pm 0.13$ according to [35] and references therein.

The relative contributions to $F_{\pi}$ and $L_{10}$ from the gluon and the quark condensates are consistent with previous evaluation $[36,37]$.

\section{Conclusions}

There have been several models describing QCD properties of the two point vectorial and axial Green function. Already an interpolation among low energy chiral properties and perturbative QCD is good [2]. The intermediate region could be phenomenologically matched with a tower of resonance states equally spaced (à la Regge) [24, 5, 27, 7]. Indeed, there are excellent dynamical reasons that Regge trajectories are dynamically generated. Holographic QCD gives us a fundamental theoretical tool with the SW model to start directly from a theory where Regge trajectories are analytically implemented. The original model [18] had several difficulties: the absence of a satisfying description of chiral symmetry breaking and of the axial sector, wrong OPE. For some further attempts to cure these problems one can refer to [40, 39].

It is natural to modify opportunely the SW model to comply with the OPE. While it was done already for the HW model [?, 37], it is a novelty for the vector correlator in the SW model: we obtain the solution for the vector field $f_{V}$ profile and the analytic expression $\Pi_{V}\left(Q^{2}\right)$ in eq. (2.20) in terms of the Digamma function $\psi$ and its derivatives. As a result, we support previous works $[24,27,38]$.

The coefficients of the polynomial of the dilaton profile $\left(b_{k}\right.$ 's), fixed by the requirement of a correct vectorial OPE, appear also in the differential equation (2.25) of the axial vector field

\footnotetext{
${ }^{1}$ Let us notice that if we had made the choice for $\sigma \simeq 0.9 \mathrm{GeV}^{2}$ as in [18] the values obtained would have been $F_{\pi} \simeq 80 \mathrm{MeV}$ and $10^{3} L_{10} \simeq-6.2$ which remain quite acceptable too.
} 
$f_{A}$, whose expression extends the analogous ones in Ref. $[14,11,28,12]$ with the presence of a scalar field in the bulk with a non-trivial vacuum profile, $v(z)$. A coherent, complete and phenomenologically consistent solution emerges: due the phenomenologically observed relation $8 \kappa^{2}=M_{a 1}^{2}$ the solution for the axial in eq. (2.25) can be obtained from the vectorial one as $\Pi_{A}\left(Q^{2}\right)=\Pi_{V}\left(Q^{2}+4 \kappa^{2}\right)+$ "corrections". This solution generates the pion pole, the correct axial spectrum and axial OPE, if the profile of the vacuum, $v(z)$, is not only a polynomial but contains also a Dirac $\delta$-function term, i.e. a boundary term for the axial field, which is needed in order to comply with the axial OPE.

Our analytic solution for $F_{\pi}$ and $L_{10}$ respectively in eq. (3.4) and (3.6) are very successfully phenomenologically and show how these parameter are linked to Regge spacing and QCD condensates; compared to previous literature [24, 5, 27, 7] our results are fully analytical.

\section{Acknowledgements}

D.G. wants to thank the organizers to have given the nice opportunity to present this work. G. D'A. acknowledges partial support by MIUR under project 2010YJ2NYW. L.C. and D.G. were supported in part by MIUR under project 2010YJ2NYW and by INFN research initiative PhenoLNF.

\section{References}

[1] L. Cappiello, G. D’Ambrosio and D. Greynat, "Assuming Regge trajectories in holographic QCD: from OPE to Chiral Perturbation Theory", Eur. Phys. J. C 75, no. 10, 465 (2015)

[2] S. Peris, M. Perrottet and E. de Rafael, "Matching long and short distances in large N(c) QCD", JHEP 9805, 011 (1998)

[3] P.D.B. Collins, "Regge theory and particle physics", Phys. Reports 1 (1971) 103.

[4] G. 't Hooft, Nucl. Phys. B75 (1974)461;

C.G. Callan et al., Phys. Rev. D13 (1976) 1649;

M.B. Einhorn, Phys. Rev. D14 (1976) 3451.

[5] M. Golterman and S. Peris, "Large N(c) QCD meets Regge theory: The Example of spin one two point functions" JHEP 0101, 028.

[6] A. B. Kaidalov, "Regge poles in QCD", In Shifman, M. (ed.): At the frontier of particle physics, vol. 1 603-636

[7] M. Shifman and A. Vainshtein, "Highly Excited Mesons, Linear Regge Trajectories and the Pattern of the Chiral Symmetry Realization", Phys. Rev. D 77, 034002 (2008)

[8] J. M. Maldacena, "The large N limit of superconformal field theories and supergravity", Adv. Theor. Math. Phys. 2, 231 (1998).

[9] S. S. Gubser, I. R. Klebanov and A. M. Polyakov, "Gauge theory correlators from noncritical string theory", Phys. Lett. B 428, 105 (1998)

[10] E. Witten, "Anti-de Sitter space and holography", Adv. Theor. Math. Phys. 2, 253 (1998)

[11] J. Erlich, E. Katz, D. T. Son and M. A. Stephanov, "QCD and a holographic model of hadrons", Phys. Rev. Lett. 95, 261602 (2005) 
[12] L. Da Rold and A. Pomarol, "Chiral symmetry breaking from five dimensional spaces", Nucl. Phys. B 721, 79 (2005)

[13] J. Hirn and V. Sanz, "Interpolating between low and high energy QCD via a 5-D Yang-Mills model", JHEP 0512, 030 (2005)

[14] D. T. Son and M. A. Stephanov, "QCD and dimensional deconstruction", Phys. Rev. D 69, 065020 (2004)

[15] S. J. Brodsky, G. F. de Teramond, H. G. Dosch and J. Erlich, "Light-Front Holographic QCD and Emerging Confinement", arXiv:1407.8131 [hep-ph].

[16] A. A. Migdal, "Series Expansion for Mesonic Masses in Multicolor QCD", Annals Phys. 110, 46 (1978).

[17] M. Shifman, "Highly excited hadrons in QCD and beyond", hep-ph/0507246.

[18] A. Karch, E. Katz, D. T. Son and M. A. Stephanov, "Linear confinement and AdS/QCD", Phys. Rev. D 74, 015005 (2006).

[19] O. Andreev, "1/q**2 corrections and gauge/string duality", Phys. Rev. D 73, 107901 (2006)

[20] O. Cata, "Towards understanding Regge trajectories in holographic QCD", Phys. Rev. D 75, 106004 (2007)

[21] F. Zuo and T. Huang, "Comments on the two-dimensional power correction in the soft wall model", Chin. Phys. Lett. 25, 3601 (2008)

[22] C. Csaki and M. Reece, "Toward a systematic holographic QCD: A Braneless approach", JHEP 0705, 062 (2007)

[23] G. Veneziano, "Construction of a crossing - symmetric, Regge behaved amplitude for linearly rising trajectories", Nuovo Cim. A 57, 190 (1968).

[24] M. Golterman, S. Peris, B. Phily and E. De Rafael, "Testing an approximation to large N(c) QCD with a toy model", JHEP 0201, 024 (2002)

[25] C. A. Dominguez, "Pion form-factor in large N(c) QCD", Phys. Lett. B 512, 331 (2001)

[26] S. S. Afonin, A. A. Andrianov, V. A. Andrianov and D. Espriu, "Matching Regge theory to the OPE", JHEP 0404, 039 (2004)

[27] O. Cata, M. Golterman and S. Peris, "Duality violations and spectral sum rules", JHEP 0508, 076 (2005).

[28] H. J. Kwee and R. F. Lebed, "Pion Form Factor in Improved Holographic QCD Backgrounds", Phys. Rev. D 77, 115007 (2008)

[29] G. 't Hooft, "A Planar Diagram Theory for Strong Interactions", Nucl. Phys. B72 (1974) 461.

[30] E. Witten, "Baryons in the 1/N Expansion", Nucl. Phys. B160 (1979) 57.

[31] J. Beringer et al. [Particle Data Group Collaboration], "Review of Particle Physics (RPP)", Phys. Rev. D 86, 010001 (2012).

[32] P. Masjuan, E. Ruiz Arriola and W. Broniowski, "Systematics of radial and angular-momentum Regge trajectories of light non-strange $q \bar{q}$-states", Phys. Rev. D 85, 094006 (2012)

[33] M. Shifman, A. Vainshtein and V. Zakharov, "QCD and Resonance Physics. The rho-omega Mixing", Nucl. Phys. B147 (1979) 385; 447. 
[34] P. Cox and T. Gherghetta, "A Soft-Wall Dilaton", JHEP 1502 (2015) 006

[35] G. Ecker, "Facets of chiral perturbation theory", Nucl. Phys. Proc. Suppl. 245 (2013) 1

[36] E. de Rafael, "Chiral Lagrangians and kaon CP violation", In *Boulder 1994, Proceedings, CP violation and the limits of the standard model* 15-84, and Marseille Cent. Theor. Phys. - 95-P-3161 (95/01,rec.Feb.) 71 p. (507515)

[37] J. Hirn, N. Rius and V. Sanz, "Geometric approach to condensates in holographic QCD", Phys. Rev. D 73, 085005 (2006)

[38] M. A. Shifman, Quark-hadron duality. Published in the Boris Ioffe Festschrift 'At the Frontier of Particle Physics Handbook of QCD', ed. M. Shifman (World Scientific, Singapore, 2001).

[39] B. Batell and T. Gherghetta, "Dynamical Soft-Wall AdS/QCD", Phys. Rev. D 78, 026002 (2008)

[40] G. F. de Teramond and S. J. Brodsky, "Light-Front Holography and Gauge/Gravity Duality: The Light Meson and Baryon Spectra", Nucl. Phys. Proc. Suppl. 199, 89 (2010) 